\title{
Quatitative Comparison of EEG Compressed Sensing using Gabor and K-SVD Dictionaries
}

\author{
Phuong Thi Dao, Xue Jun Li and Anthony Griffin \\ Department of Electrical and Electronic Engineering \\ Auckland University of Technology \\ Auckland, New Zealand \\ \{pdao, xuejun.li, anthony.griffin\}@aut.ac.nz
}

\begin{abstract}
With the fast development of wearable healthcare systems, compressed sensing (CS) has been proposed to be applied in electroencephalogram (EEG) acquisition. For CS, it is desired to build the best-fit dictionary in order to achieve good reconstruction accuracy. While most of existing works focused on static dictionaries such as Gabor, Fourier and wavelets, the dynamic nature of EEG signals motivates us to study learned dictionaries, which are supposed to provide better reconstruction accuracy and lower computation cost. In this paper, we provide the quantitative performance comparison of EEG CS using two different types of dictionaries, i.e., the well-known Gabor dictionaries versus K-SVD learned dictionaries. The performance comparison utilizes the well-established database of scalp EEG from Physiobank, which allows researchers in this field to compare their work with ours. In addition, it also attempts to inspire the systematic study of dictionary learning in EEG CS.
\end{abstract}

Keywords-Electroencephalogram signal, compressed sensing, compression ratio, Gabor, K-SVD, dictionary learning.

\section{INTRODUCTION}

Compression of electrical biosignals, such as electrocardiogram (ECG), electroencephalogram (EEG) [1], electromyogram (EMG) has been an interesting research topic [2]. Compressed sensing (CS) [3] recently emerged as a promising technique that allows for higher compression ratios at an acceptable level of distortion, albeit at a low cost of power consumption. In addition, with the benefit of minimizing the data volume and energy utilization in the sensor node, CS is best fitted with mobile and wearable telemedicine systems.

$\mathrm{CS}$ is based on the fundamental fact that sparse signals can be represented using only a few non-zero coefficients in a suitable dictionary [4]. In the compression stage, the compressed signal is obtained as the product of the input signal and the sparse sensing matrix. Since the number of compressive measurements taken is far less than the number of samples in the original signal, the major difficulty is how to reconstruct the original signal from the compressed signal. This task is usually achieved by convex optimization algorithms such as Basis Pursuit (BP) or the Orthogonal Matching Pursuit (OMP) with the utilization of the sparse dictionary [5].

For signals that are not sparse in time domain or transform domain, e.g., EEG signals, it is desirable to identify a suitable dictionary that is able to sparsely represent the signal before applying the optimization solution.
Among the time-frequency transforms techniques such as Fourier and wavelet transforms, Gabor has emerged as the best representation of EEG signals in both time and frequency domain [6-8]. As a result, most of the current work in EEG compressed sensing focus on building a Gabor dictionary [5, 9, 10]. Given the fact that a Gabor dictionary is an over-complete dictionary, it can achieve greater robustness in the presence of noise. In addition, it can be sparser and can have greater flexibility in matching structure. Consequently, it can achieve a more accurate reconstruction [11]. However, a Gabor dictionary has difficulty reconstructing complex waveforms such as spikeand-wave and/or polyspike-and-wave EEG.

In an attempt to increase the flexibility of the dictionary to best fit the natural signals, Tosic and Frossard introduced a novel technique that utilized real signals to learn a data-driven dictionary [12]. Inspired by their work, dictionary-learning methods have been proposed in biomedical signal such as ECG, EEG and EMG. While most of the existing works focus on ECG $[13,14]$ signals, there are fewer studies reported using EEG signals. Moreover, event-related EEG signals used in BrainComputer-Interface $(\mathrm{BCI})$ tend to be the potential candidate signals. To the best of our knowledge, there is only one study that applied dictionary learning to clinical EEG signals with CS [15]. This study considered the signal segments that contain only one spike in order to simplify the performance evaluation process. Moreover, detailed information about the adopted EEG database was missing.

Enthused by the existing work of dictionary learning on EEG compression, we attempt to perform a systematic study of dictionary learning in clinical EEG with CS. We start with a quantitative performance comparison of EEG CS in two different types of dictionaries-well-known Gabor dictionaries and emerging K-SVD learned dictionaries. The CHB-MIT scalp EEG database $[16,17]$ is the most popular and its free online access makes it easy for worldwide researchers to compare their results with ours.

The rest of this paper is organized as follows. Section II revisits the background EEG compression with CS. Section III describes our methods to set up the Gabor and K-SVD dictionaries. Section IV presents the experimental setup. Section $\mathrm{V}$ discusses the results and Section VI concludes the paper with our findings. 


\section{EEG COMPRESSED SESNSING}

Given a sparse signal $\mathbf{x} \in \mathbb{R}^{N \times 1}$, it can be compressed by a random matrix $\boldsymbol{\Phi} \in \mathbb{R}^{M \times N}$ with compressed sensing:

$$
\mathbf{y}=\mathbf{\Phi} \mathbf{x}
$$

where $\mathbf{x}$ is the input signal, $\mathbf{\Phi}$ is the sampling matrix, and $\mathbf{y}$ is the compressed signal containing $M$ measurements. The compression is achieved by setting $M \ll N$.

The measurement matrix $\boldsymbol{\Phi}$ must be maximally incoherent with the chosen dictionary. Previous works have shown that random matrices such as Gaussian, Bernoulli and sparse binary sensing matrices satisfy this requirement [9] [11] [18] [19].

Given that the input signal is sparse in the dictionary $\mathbf{D} \in \mathbb{R}^{N \times P}$, we can represent the signal $\mathbf{x}$ as

$$
\mathbf{x}=\mathbf{D} \boldsymbol{\theta}=\sum_{i=1}^{K} \boldsymbol{\theta}_{\mathbf{i}} \mathbf{d}_{\mathbf{i}}
$$

where $K$ is the number of non-zero elements in the coefficient vector $\boldsymbol{\theta}$, and the signal $\mathbf{x}$ can be represented by a few atoms $\mathbf{d}_{\mathbf{i}}$ from the dictionary.

Thus, the compressed signal can be obtained by:

$$
\mathbf{y}=\Phi \mathbf{x}=\Phi \mathbf{\Phi}
$$

The fundamental idea behind CS is that we can represent signals using only a few non-zero coefficients in a sparse dictionary. In this paper, we mainly focus on performance of compressed sensing in EEG signals using static Gabor dictionaries and K-SVD learned dictionaries.

Given the compressed signal $\mathbf{y}$, the reconstruction stage needs to find $\mathbf{x}$ in order to satisfy (3). As the length of the unknowns $\mathbf{x}$ and $\boldsymbol{\theta}$ is much larger than the length of $\mathbf{y}$, there are infinite solutions for this equation.

The classical solution for this type of inverse problem is to minimize the Euclidean norm of the approximate signal $\hat{\mathbf{x}}$ subject to satisfying that the product of the measurement matrix $\boldsymbol{\Phi}$ with the reconstructed signal is equal to the compressed signal $\mathbf{y}$. This solution is called least squares minimization or $\ell_{2}$ norm minimization:

$$
\tilde{\mathbf{x}}=\arg \min \|\hat{\mathbf{x}}\|_{2} \text { s.t. } \boldsymbol{\Phi} \hat{\mathbf{x}}=\mathbf{y}
$$

Although this equation has a convenient closed form solution given by the normal equation, the solution is almost never sparse, resulting in a high reconstruction error.

As the $\ell_{2}$ norm minimization does not usually return a sparse vector, alternatives have been sought. One is to directly enforce a sparsity constraint on the solution, namely the $\ell_{0}$ norm, to minimize the number of non-zero elements in $\hat{\mathbf{x}}$ as:

$$
\tilde{\mathbf{x}}=\arg \min \|\hat{\mathbf{x}}\|_{0} \text { s.t. } \boldsymbol{\Phi} \hat{\mathbf{x}}=\mathbf{y}
$$

However this is computationally unfeasible as it is NP hard. The solution is to relax (5) and use the $\ell_{1}$ norm, thereby minimizing the magnitude of the non-zero elements in $\hat{\mathbf{x}}$, so (5) becomes

$$
\tilde{\mathbf{x}}=\arg \min \|\hat{\mathbf{x}}\|_{1} \text { s.t. } \boldsymbol{\Phi} \hat{\mathbf{x}}=\mathbf{y}
$$

The $\ell_{1}$ norm has been proved to achieve a unique solution under certain conditions. As a result, $\mathbf{x}$ can be reconstructed exactly from compressed signal $\mathbf{y}$.

With the constraint that $\mathbf{x}$ is sparse and the knowledge of the dictionary $\mathbf{D}$, the solution of $\mathbf{x}$ is equivalent to finding the best fit for the coefficient vector $\boldsymbol{\theta}$. As a result, the problem now becomes minimizing the magnitude of the non-zero elements in $\boldsymbol{\theta}$ :

$$
\min \|\boldsymbol{\theta}\|_{1} \text { s.t. } \mathbf{y}=\boldsymbol{\Phi D} \boldsymbol{\theta}
$$

\section{METHODS}

As shown in Figure 1, the EEG signal is first segmented into non-overlapping sections of length $N=1024$ (4-second). The mean will be removed before processing to enhance the signal sparsity. This mean must be stored for restoring in the reconstruction stage.

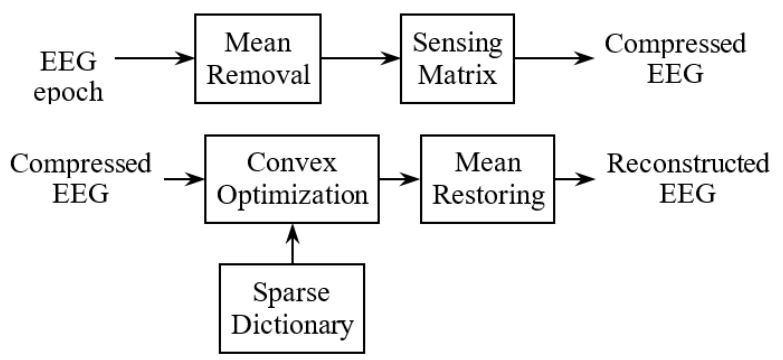

Figure 1. Flowchart of EEG CS

\section{A. Compression}

In the compression stage, the choice of sensing matrix ensures the maximal incoherent characteristics of the EEG signal without the knowledge of dictionary. We selected the white-noise Gaussian random matrix [20] as our sensing matrix to minimize computation cost.

\section{B. Reconstruction}

Selecting the best matching atoms from the over-complete dictionary to reconstruct the sparse representation is essential in solving the $\ell_{1}$ norm minimization in (7). Algorithms such as Matching Pursuit (MP), Orthogonal Matching Pursuit (OMP) and Basic Pursuit (BP) have been proposed to improve the reconstruction efficiency $[9,11,18,19,21-23]$. 
Fauvel and Rabab [24] noted that the Basis pursuit de-noise algorithm (BPDN) required fewer measurements than other greedy algorithms to achieve a comparable reconstruction quantity. Moreover, BPDN is packed in Matlab solver for a large-scale one-norm regularized least squares named SPGL1 [25]. For these reasons, we selected BPDN as our reconstruction algorithm.

BPDN attempts to solve the regularization problem with a trade-off between having a small residual $\sigma$ and making $\theta$ simple in the norm-1 sense:

$$
\min \|\boldsymbol{\theta}\|_{1} \text { s.t. }\|\mathbf{y}-\boldsymbol{\Phi D} \boldsymbol{\theta}\|_{2}^{2}<\sigma
$$

By varying the value of $\sigma$ and performing the test on a dataset of 100 random selection EEG segments as shown in Figure 2, we thus chose $\sigma=0.01$.

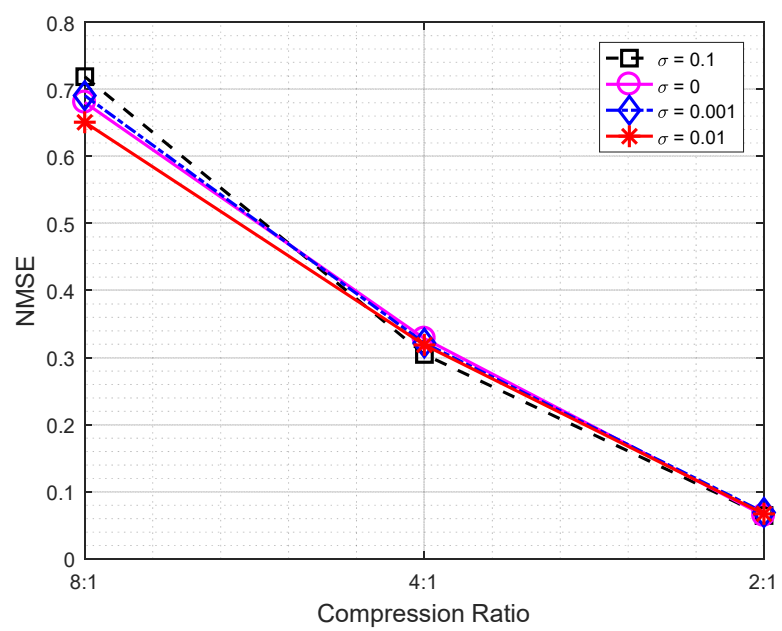

(a) Mean NMSE vs. CR

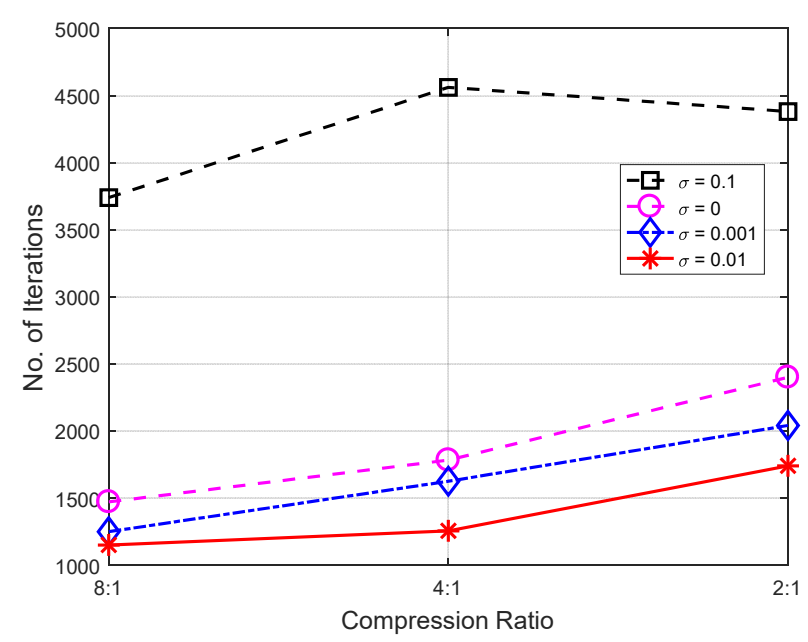

(b) number of iteration to reach BPDN solution vs. CR

Figure 2. Performance of BPDN at different $\mathrm{CR}$ values

\section{Static Gabor Dictionary Contruction}

Gabor dictionary atoms are parameterized as:

$$
g\left(n ; n_{0}, f_{0}, s\right)=K_{\left(n_{0}, f_{0}, s\right)} \exp \left[-\frac{\left(n-n_{0}\right)^{2}}{2 s^{2}}\right] \sin \left[2 \pi f_{0}\left(n-n_{0}\right)\right]
$$

where $s$ is the scale of atom, $\mathrm{n}_{0}$ and $\mathrm{f}_{0}$ are the center of atom, $K\left(n_{0}, f_{0}, s\right)$ is the normalization factor.

The time and frequency steps decide the size of dictionary and play a vital role in the reconstruction stage. Inspired by the work of Simon Fauvel et.al $[19,26]$, we define $t_{f}$ and $f_{f}$ as time and frequency factors and obtain the time and frequency step base on the following equation:

$$
\Delta f_{0}=\frac{2 \sqrt{2 \pi \alpha}}{s B f_{f}}, \Delta n_{0}=s B t_{f} \sqrt{\frac{2 \alpha}{\pi}}
$$

where $\alpha=0.5 \ln \left[0.5\left(B+\frac{1}{B}\right)\right]$ and $\mathrm{B}=2$ is the base.

\section{K-SVD Dictionary Learning}

Different from a fixed Gabor dictionary, dictionary-learning approaches proposed a data-driven method to obtain a signal adapted dictionary. The well-known K-SVD dictionary learning method [19, 27, 28] based on generalizing the K-mean method by extend the sparsity constrains to a linear combination of atoms in the dictionary. Initializing with a set of real signals, the K-SVD method works by iteratively alternating between sparse coding and updating the dictionary atoms with an attempt to fit the data. K-SVD is flexible, works well with any pursuit method and takes advantage of both the spatial and temporal redundancy of multi-channel EEG signals [29].

\section{EXPERIMENTAL SETUP}

\section{A. Database Selection}

Theoretically, any standard EEG signal is suitable for a compressed sensing system. However, the diversity in selecting EEG databases has made it hard for researchers to compare their algorithms with previous approaches. To make it possible for worldwide researchers to verify and compare their works, an online available EEG database is the best choice. The most commonly-used EEG signals are from the Neuroelectric Database from PhysioBank [14], EPILEPSIAE database from University of Freiburg [30] and the database from the BCI Competition [25].

While the other two databases focus on clinical EEG signals, the database from BCI Competition focused on the EEG signals corresponding to a specific mental state from healthy subjects.

The largest and most comprehensive EEG database, which is suitable for a broad band of application, is EPILEPSIAE. Up to 122 EEG channels from 200 patients with epilepsy was collected for a continuous recording time at least 96 hours at a sample rate up to $2500 \mathrm{~Hz}$. This results with the huge database up to thousands of hours in total. Unfortunately, it is not a free downloadable database.

CHB-MIT scalp EEG database, which is freely accessible 
from PhysioBank is a big database of up to several days recording with well-defined seizure notation. The database was collected up to 23 EEG channels at the Children's Hospital Boston from 24 paediatric subjects with intractable seizures. There are a total of 664 .edf files collected at a sampling frequency of $256 \mathrm{~Hz}$ and a resolution of 16 bits per sample.

For research purposes, we strongly recommend using the CHB-MIT database to check the performance of EEG compressed sensing approaches.

\section{B. Data Collection}

In total, 11,200 non-overlapping segments of 4-second EEG signals were randomly chosen from the database. Note that not all channels are EEG signals. ECG may simultaneously be recorded in conjunction with the EEG and some specific EEG channels may be omitted in some cases for diagnosis.

In this paper, we used 3,200 segments for testing and thus left 8,000 segments for the dictionary training.

\section{Performance Metrics}

Given $N$ is the length of original EEG signal, $\mathrm{M}$ is the length of compressed signal, the compression ratio is defined by $C R=N / M$. The accuracy of reconstruction signal is evaluated by the normalized mean square error (NMSE) as

$$
\operatorname{NMSE}(\mathbf{x}, \hat{\mathbf{x}})=\frac{\|\mathbf{x}-\hat{\mathbf{x}}\|}{\left\|\mathbf{x}-\boldsymbol{\mu}_{\mathbf{x}}\right\|}
$$

where $\mathbf{x}$ is the original signal, $\hat{\mathbf{x}}$ is the reconstructed signal and $\boldsymbol{\mu}_{\mathbf{x}}$ is the mean of $\mathbf{x}$.

\section{RESULTS AND DisCUSSION}

A set of nine Gabor dictionaries were built and nine K-SVD dictionaries were learned to check the performance of the testing signals at the compression ratio values 2,4 and 8 .

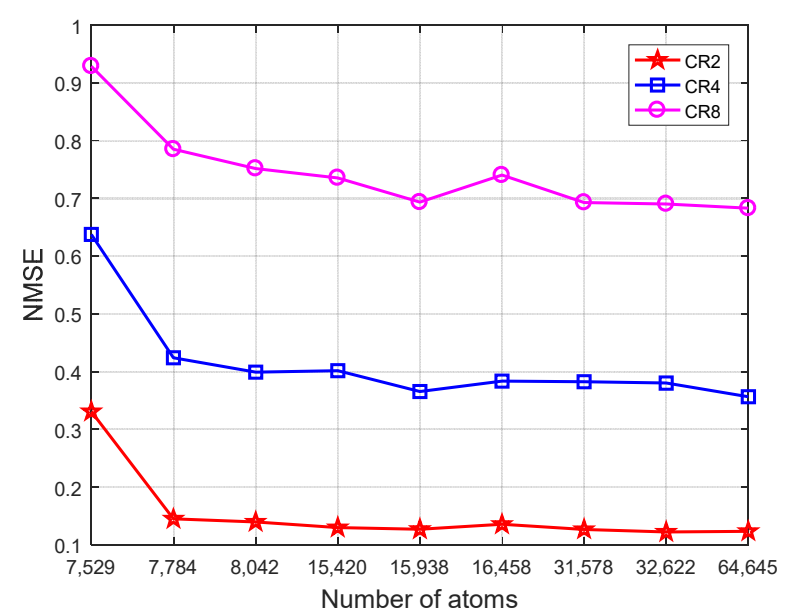

(a) Gabor dictionaries

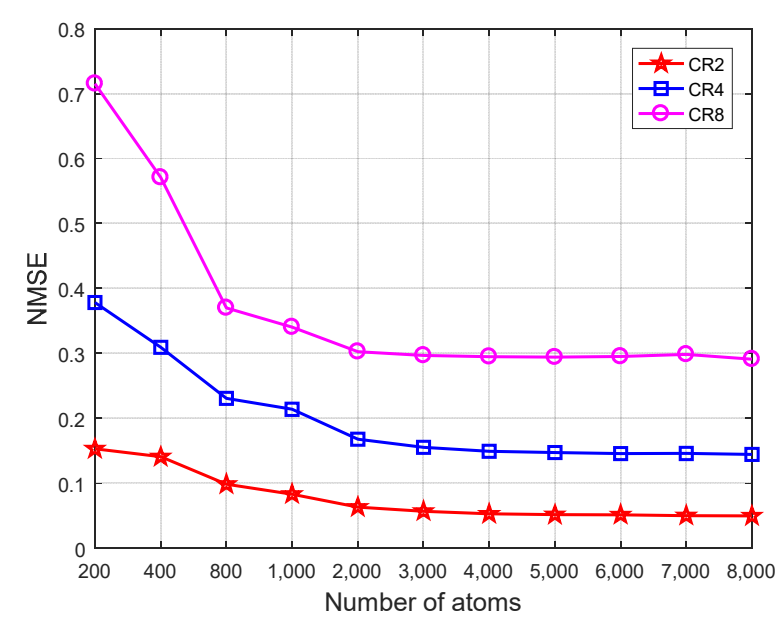

(b) K-SVD dictionaries

Figure 3. Mean NMSE vs. size of dictionaries for (a) Gabor and (b) K-SVD

Figure 3 shows the mean NMSE versus the size of chosen dictionaries. It is very clear from the plot that even though the NMSE improves by increasing the dictionary size, the effect provides diminishing returns, particularly above a Gabor dictionary size of 15,938 and the K-SVD dictionary size of 3,000 atoms. These two dictionaries seem to be the best choice for reconstruction performance whilst minimizing the dictionary size, given that the dictionary size will impact on memory requirements and reconstruction speed.

Figure 4 shows the comparison of mean NMSE and the reconstruction time versus the compression ratio for the chosen Gabor and K-SVD dictionary. It is obviously that with a 5-fold size decrease, the K-SVD dictionary still achieves a far better NMSE than the Gabor dictionary at CR of 4:1 and above. As a result, it is more beneficial to focus on learning a dictionary than continuing with a static dictionary. The future work will focus on selecting good EEG training sets and optimal parameters sets for training. EEG signals with specific clinical condition such as seizure will be included to learn the optimal dictionary for EEG with a seizure compressed sensing system.

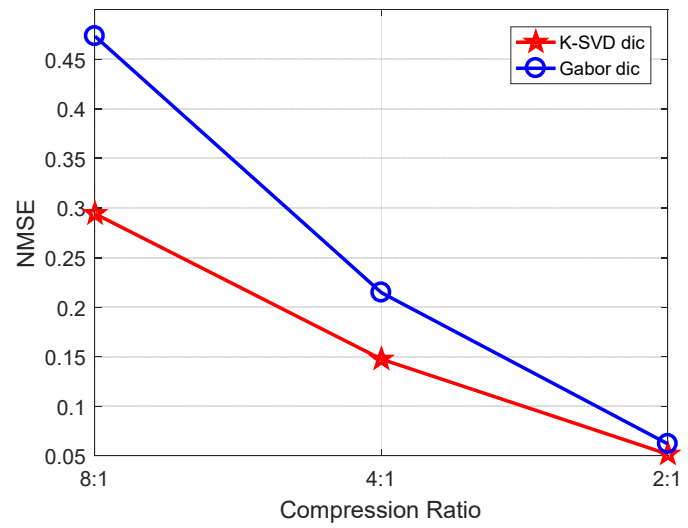

(a) NMSE 


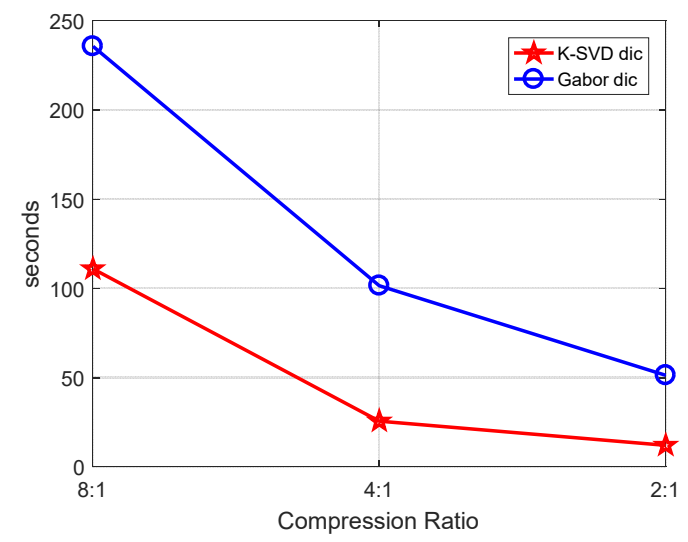

(b) Computation cost

Figure 4. (a) mean NMSE and (b) computation cost vs. dictionaries

\section{CONCLUSION}

This paper presented a systematic study of the performance comparison of EEG compressed sensing using static Gabor dictionaries and learned K-SVD dictionaries. Experimental results with CHB-MIT scalp EEG database showed that K-SVD can help to achieve a better compression ratio, lower reconstruction error and lower computational complexity. At a compression ratio of 4 , the mean NMSE values achieved by KSVD and Gabor dictionaries are 0.15 and 0.21 , respectively. This indicates that learned dictionaries should be further studied to achieve better EEG compression performance. Our future work on this topic is to investigate how other dictionary learning algorithms can help develop EEG compressed sensing for mobile telemedicine systems.

\section{REFERENCES}

[1] G. Antoniol and P. Tonella, "EEG data compression techniques," IEEE Transactions on Biomedical Engineering, vol. 44, pp. 105-114, 1997.

[2] L. Brechet, M. F. Lucas, C. Doncarli, and D. Farina, "Compression of biomedical signals with mother wavelet optimization and best-basis wavelet packet selection," IEEE Transactions on Biomedical Engineering, vol. 54, pp. 2186-2192, Dec. 2007.

[3] D. L. Donoho, "Compressed sensing," IEEE Transactions on Information Theory, vol. 52, pp. 1289-1306, April 2006.

[4] S. Aviyente, "Compressed sensing framework for EEG compression," in IEEE SSP'07, Madison, WI, USA, 2007, pp. 181-184.

[5] R. Kuś, P. T. Różański, and P. J. Durka, "Multivariate matching pursuit in optimal Gabor dictionaries: theory and software with interface for EEG/MEG via Svarog," BioMedical Engineering OnLine, vol. 12, pp. 1$28,2013$.

[6] S. Blanco, C. E. D'Attellis, S. I. Isaacson, O. A. Rosso, and R. O. Sirne, "Time-frequency analysis of electroencephalogram series. II. Gabor and wavelet transforms," Physical Review E, vol. 54, pp. 6661-6672, 1996.

[7] S. V. Schönwald, G. J. L. Gerhardt, E. L. de Santa-Helena, and M. L. F. Chaves, "Characteristics of human EEG sleep spindles assessed by Gabor transform," Physica A: Statistical Mechanics and its Applications, vol. 327, pp. 180-184, 2003.

[8] L. Chen, E. Zhao, D. Wang, Z. Han, S. Zhang, and X. Cuiping, "Feature extraction of EEG signals from epilepsy patients based on Gabor
Transform and EMD Decomposition," in 2010 Sixth International Conference on Natural Computation, 2010, pp. 1243-1247.

[9] M. Mohsina and A. Majumdar, "Gabor based analysis prior formulation for EEG signal reconstruction," Biomedical Signal Processing and Control, vol. 8, pp. 951-955, 2013.

[10] W. Yanling, Z. Hongxin, W. Haiqing, and L. Yinghua, "The sparse decomposition and compression of ECG and EEG based on matching pursuits," in Proc. of BMEI'10, 2010, pp. 1094-1097.

[11] A. Abdulghani, A. Casson, and E. Rodriguez-Villegas, "Compressive sensing scalp EEG signals: implementations and practical performance," Medical \& Biological Engineering \& Computing, vol. 50, pp. 1137-1145, 2012.

[12] I. Tosic and P. Frossard, "Dictionary Learning," Signal Processing Magazine, IEEE, vol. 28, pp. 27-38, 2011.

[13] B. Mailhe, R. Gribonval, F. Bimbot, M. Lemay, P. Vandergheynst, and J. M. Vesin, "Dictionary learning for the sparse modelling of atrial fibrillation in ECG signals," in Proc. of ICASSP'09, 2009, pp. 465-468.

[14] L. Seungjae, L. Jun, and P. H. Chou, "A new approach to compressing ECG signals with trained overcomplete dictionary," in Proc. of Mobihealth'14, 2014, pp. 83-86.

[15] J. Zhang, Y. Suo, S. Mitra, S, S. Hsiao, R. F. Yazicioglu, et al., "An Efficient and Compact Compressed Sensing Microsystem for Implantable Neural Recordings," IEEE Transactions on Biomedical Circuits and Systems, vol. 8, pp. 485-496, 2014.

[16] A. L. Goldberger, et al., "PhysioBank, PhysioToolkit, and PhysioNet component of a new research resource for complex physiologic signals," Circulation Electronic Pages, 2002.

[17] "EEG Database at http://www.physionet.org."

[18] Z. Zhilin, J. Tzyy-Ping, S. Makeig, and B. D. Rao, "Compressed Sensing of EEG for Wireless Telemonitoring With Low Energy Consumption and Inexpensive Hardware," IEEE Transactions on Biomedical Engineering, vol. 60, pp. 221-224, 2013.

[19] S. Fauvel and R. Ward, "An Energy Efficient Compressed Sensing Framework for the Compression of Electroencephalogram Signals," Sensors, vol. 14, 2014.

[20] D. Craven, B. McGinley, L. Kilmartin, M. Glavin, and E. Jones, "Compressed Sensing for Bioelectric Signals: A Review," IEEE Journal of Biomedical and Health Informatics, vol. 19, pp. 529-540, 2015.

[21] J. A. Tropp, A. C. Gilbert, and M. J. Strauss, "Simultaneous sparse approximation via greedy pursuit," in Proc. of ICASSP'05, 2005.

[22] S. S. Chen, D. L. Donoho, and M. A. Saunders, "Atomic Decomposition by Basis Pursuit," SIAM Journal on Scientific Computing, vol. 20, pp. 3361, 1998.

[23] S. G. Mallat and Z. Zhang, "Matching pursuits with time-frequency dictionaries," IEEE Transactions on Signal Processing, vol. 41, pp. 3397 3415, 1993.

[24] Y. C. Pati, R. Rezaiifar, and P. S. Krishnaprasad, "Orthogonal matching pursuit: recursive function approximation with applications to wavelet decomposition," in Proc. of ACSSC'93, 1993, pp. 40-44 vol.1.

[25] Q. Barthélemy, C. Gouy-Pailler, Y. Isaac, A. Souloumiac, A. Larue, and J. I. Mars, "Multivariate temporal dictionary learning for EEG," Journal of Neuroscience Methods, vol. 215, pp. 19-28, 2013.

[26] M. Aharon, M. Elad, and A. M. Bruckstein, "On the uniqueness of overcomplete dictionaries, and a practical way to retrieve them," Linear Algebra and its Applications, vol. 416, pp. 48-67, 2006.

[27] E. v. d. Berg and M. P. Friedlander, "Probing the Pareto Frontier for Basis Pursuit Solutions," SIAM Journal on Scientific Computing, vol. 31, pp. 890-912, 2009.

[28] M. Aharon, M. Elad, and A. Bruckstein, "K-SVD: An Algorithm for Designing Overcomplete Dictionaries for Sparse Representation," IEEE Transactions on Signal Processing, vol. 54, pp. 4311-4322, 2006.

[29] L. N. Smith and M. Elad, "Improving Dictionary Learning: Multiple Dictionary Updates and Coefficient Reuse," IEEE Signal Processing Letters, vol. 20, pp. 79-82, 2013.

[30] O. Bryt and M. Elad, "Compression of facial images using the K-SVD algorithm," Journal of Visual Communication and Image Representation, vol. 19 , pp. $270-282,2008$. 